Bull. Korean Math. Soc. 41 (2004), No. 1, pp. 53-71

\title{
ON A SUBCLASS OF CERTAIN STARLIKE FUNCTIONS WITH NEGATIVE COEFFICIENTS
}

\author{
Muhammet Kamali and Halit Orhan
}

\begin{abstract}
A certain subclass $T_{\Omega}(n, p, \lambda, \alpha)$ of starlike functions in the unit disk is introduced. The object of the present paper is to derive several interesting properties of functions belonging to the class $T_{\Omega \Omega}(n, p, \lambda, \alpha)$. Coefficient inequalities, distortion theorems and closure theorems of functions belonging to the class $T_{\Omega}(n, p, \lambda, \alpha)$ are determined. Also we obtain radii of convexity for the class $T_{\Omega}(n, p, \lambda, \alpha)$. Furthermore, integral operators and modified Hadamard products of several functions belonging to the class $T_{\Omega}(n, p, \lambda, \alpha)$ are studied here.
\end{abstract}

\section{Introduction}

Let $A$ be class of functions $f(z)$ of the form $f(z)=z+\sum_{k=2}^{\infty} a_{k} z^{k}$ which are analytic in the open unit disk $U=\{z:|z|<1\}$. For $f(z)$ belong to $A$, Salagean [5] has introduced the following operator called the Salagean operator:

$$
\begin{aligned}
& D^{0} f(z)=f(z), D^{1} f(z)=D f(z)=z f^{\prime}(z) \\
& D^{n} f(z)=D\left(D^{n-1} f(z)\right)(n \in \mathrm{N}=\{1,2,3, \ldots\}) .
\end{aligned}
$$

Note that $D^{n} f(z)=z+\sum_{k=2}^{\infty} k^{n} a_{k} z^{k}, n \in \mathrm{N}_{0}=\{0\} \cup \mathrm{N}$.

Let $T(n, p)$ denote the class of functions $f(z)$ of the form:

$$
\begin{aligned}
f(z)= & z^{p}-\sum_{k=n}^{\infty} a_{k+p} z^{k+p} \\
& \left(a_{k+p} \geq 0 ; p \in \mathrm{N}:=\{1,2,3, \ldots\} ; n \in \mathrm{N}\right),
\end{aligned}
$$

which are analytic in the open unit disk $U=\{z \in \mathrm{C}:|z|<1\}$.

Received August 26, 2002.

2000 Mathematics Subject Classification: Primary 30C45.

Key words and phrases: Starlike functions, Hadamard product, Radii of convexity, Salagean operator, p-valent. 
A function $f(z) \in T(n, p)$ is said to be in the class $T(n, p, \lambda, \alpha)$ if it satisfies the inequality:

$$
\operatorname{Re}\left\{\frac{z f^{\prime}(z)+\lambda z^{2} f^{\prime \prime}(z)}{(1-\lambda) f(z)+\lambda z f^{\prime}(z)}\right\}>\alpha
$$

for some $\alpha \quad(0 \leq \alpha<1)$ and $\lambda \quad(0 \leq \lambda \leq 1)$, and for all $z \in U$ [2].

We can write the following equalities for the functions $f(z)$ belong to the class $T(n, p)$

$$
\begin{aligned}
D^{0} f(z) & =f(z) \\
D^{1} f(z) & =D f(z)=z f^{\prime}(z)=z\left[p z^{p-1}-\sum_{k=n}^{\infty}(k+p) a_{k+p} z^{k+p-1}\right] \\
& =p z^{p}-\sum_{k=n}^{\infty}(k+p) a_{k+p} z^{k+p} \\
D^{2} f(z) & =D(D f(z))=p^{2} z^{p}-\sum_{k=n}^{\infty}(k+p)^{2} a_{k+p} z^{k+p}, \\
& \vdots \\
D^{\Omega} f(z) & =D\left(D^{\Omega-1} f(z)\right)=p^{\Omega} z^{p}-\sum_{k=n}^{\infty}(k+p)^{\Omega} a_{k+p} z^{k+p} .
\end{aligned}
$$

A function $f(z) \in T(n, p)$ is said to be in the class $T_{\Omega}(n, p, \lambda, \alpha)$ if it satisfies the inequality:

$$
\operatorname{Re}\left\{\frac{(1-\lambda) z\left(D^{\Omega} f(z)\right)^{\prime}+\lambda z\left(D^{\Omega+1} f(z)\right)^{\prime}}{(1-\lambda) D^{\Omega} f(z)+\lambda D^{\Omega+1} f(z)}\right\}>\alpha \quad\left(\Omega \in \mathrm{N}_{0}\right)
$$

for some $\alpha(0 \leq \alpha<1)$ and $\lambda(0 \leq \lambda \leq 1)$, and for all $z \in U$ [2] .

We note that

$$
\begin{aligned}
T_{0}(n, p, \lambda, \alpha) & \equiv T(n, p, \lambda, \alpha), \\
T_{0}(n, 1,0, \alpha) & \equiv T_{\alpha}(n), \\
T_{0}(n, 1,1, \alpha) & \equiv C_{\alpha}(n), \\
T_{0}(1,1,0, \alpha) & \equiv T^{*}(\alpha), \\
T_{0}(1,1,1, \alpha) & \equiv C(\alpha), \\
T_{0}(n, 1, \lambda, \alpha) & \equiv P(n, \lambda, \alpha),
\end{aligned}
$$

and

$$
T_{1}(n, 1, \lambda, \alpha) \equiv C(n, \lambda, \alpha)
$$


The classes $T_{\alpha}(n)$ and $C_{\alpha}(n)$ were studied earlier by Srivastava et al. [8], the classes $T^{*}(\alpha) \equiv T_{\alpha}(1)$ and $C(\alpha) \equiv C_{\alpha}(1)$ were studied by Silverman [7], the class $P(n, \lambda, \alpha)$ was studied by Altıntas [1], the class $T(n, p, \lambda, \alpha)$ were studied by Altıntaş et al. [2], and the class $C(n, \lambda, \alpha)$ were studied by Kamali and Akbulut [4].

\section{A theorem on coefficient bounds}

We begin by proving some sharp coefficient inequalities contained in the following theorem.

TheOREM 1. A function $f(z) \in T(n, p)$ is in the class $T_{\Omega}(n, p, \lambda, \alpha)$ if and only if

$$
\begin{aligned}
& \sum_{k=n}^{\infty}(k+p)^{\Omega}(k+p-\alpha)(\lambda k+\lambda p-\lambda+1) a_{k+p} \leq p^{\Omega}(p-\alpha)(1+\lambda p-\lambda) \\
& \quad\left(0 \leq \alpha<1 ; 0 \leq \lambda \leq 1 ; p \leq p^{\Omega}(p-\alpha)(1+\lambda p-\lambda)(p \neq 1) ;\right. \\
& \left.\quad p \in \mathrm{N} ; n \in \mathrm{N} ; \Omega \in \mathrm{N}_{0}\right) .
\end{aligned}
$$

The result is sharp.

Proof. Suppose that $f(z) \in T_{\Omega}(n, p, \lambda, \alpha)$. Then we find from (1.3) that

$$
\begin{gathered}
\operatorname{Re}\left\{\frac{(1+\lambda p-\lambda) p^{\Omega+1} z^{p}-\sum_{k=n}^{\infty}(\lambda k+\lambda p+1-\lambda)(k+p)^{\Omega+1} a_{k+p} z^{k+p}}{(1+\lambda p-\lambda) p^{\Omega} z^{p}-\sum_{k=n}^{\infty}(\lambda k+\lambda p+1-\lambda)(k+p)^{\Omega} a_{k+p} z^{k+p}}\right\}>\alpha \\
\left(0 \leq \alpha<1 ; 0 \leq \lambda \leq 1 ; p \leq p^{\Omega}(p-\alpha)(1+\lambda p-\lambda)(p \neq 1) ;\right. \\
\left.p \in \mathrm{N} ; n \in \mathrm{N} ; \Omega \in \mathrm{N}_{0} ; z \in U\right) .
\end{gathered}
$$

If we choose $z$ to be real and let $z \rightarrow 1^{-}$, we get

$$
\begin{gathered}
\left\{\frac{(1+\lambda p-\lambda) p^{\Omega+1}-\sum_{k=n}^{\infty}(\lambda k+\lambda p+1-\lambda)(k+p)^{\Omega+1} a_{k+p}}{(1+\lambda p-\lambda) p^{\Omega}-\sum_{k=n}^{\infty}(\lambda k+\lambda p+1-\lambda)(k+p)^{\Omega} a_{k+p}}\right\} \geq \alpha \\
\left(0 \leq \alpha<1 ; 0 \leq \lambda \leq 1 ; p \leq p^{\Omega}(p-\alpha)(1+\lambda p-\lambda)(p \neq 1) ;\right. \\
\left.p \in \mathrm{N} ; n \in \mathrm{N} ; \Omega \in \mathrm{N}_{0}\right)
\end{gathered}
$$


or, equivalently,

$$
\begin{aligned}
& \sum_{k=n}^{\infty}(\lambda k+\lambda p+1-\lambda)(k+p)^{\Omega+1} a_{k+p} \\
& \quad-\alpha \sum_{k=n}^{\infty}(\lambda k+\lambda p+1-\lambda)(k+p)^{\Omega} a_{k+p} \\
& \leq(1+\lambda p-\lambda) p^{\Omega+1}-\alpha(1+\lambda p-\lambda) p^{\Omega} \\
& \quad\left(0 \leq \alpha<1 ; 0 \leq \lambda \leq 1 ; p \leq p^{\Omega}(p-\alpha)(1+\lambda p-\lambda)(p \neq 1) ;\right. \\
& \left.\quad p \in \mathrm{N} ; n \in \mathrm{N} ; \Omega \in \mathrm{N}_{0}\right) .
\end{aligned}
$$

Thus, we obtain

$$
\begin{aligned}
& \sum_{k=n}^{\infty}(k+p-\alpha)(k+p)^{\Omega}(\lambda k+\lambda p+1-\lambda) a_{k+p} \\
\leq & (p-\alpha) p^{\Omega}(1+\lambda p-\lambda) \\
& \left(0 \leq \alpha<1 ; 0 \leq \lambda \leq 1 ; p \leq p^{\Omega}(p-\alpha)(1+\lambda p-\lambda)(p \neq 1) ;\right. \\
& \left.p \in \mathrm{N} ; n \in \mathrm{N} ; \Omega \in \mathrm{N}_{0}\right) .
\end{aligned}
$$

Conversely, suppose that the inequality (2.1) holds true and let

$$
z \in \partial U=\{z: z \in \mathrm{C}|z|=1\} .
$$

Then we find from the definition (1.1) that

$$
\begin{aligned}
& \left|\frac{(1-\lambda) z\left(D^{\Omega} f(z)\right)^{\prime}+\lambda z\left(D^{\Omega+1} f(z)\right)^{\prime}}{(1-\lambda) D^{\Omega} f(z)+\lambda D^{\Omega+1} f(z)}-p^{\Omega}(p-\alpha)(1+\lambda p-\lambda)\right| \\
= & \mid \frac{(1+\lambda p-\lambda) p^{\Omega+1} z^{p}-\sum_{k=n}^{\infty}(\lambda k+\lambda p+1-\lambda)(k+p)^{\Omega+1} a_{k+p} z^{k+p}}{(1+\lambda p-\lambda) p^{\Omega} z^{p}-\sum_{k=n}^{\infty}(\lambda k+\lambda p+1-\lambda)(k+p)^{\Omega} a_{k+p} z^{k+p}} \\
& -p^{\Omega}(p-\alpha)(1+\lambda p-\lambda) \mid \\
\leq & \frac{\left|-(1+\lambda p-\lambda) p^{\Omega}\left\{p^{\Omega}(p-\alpha)(1+\lambda p-\lambda)-p\right\} z^{p}\right|}{\left|\sum_{k=n}^{\infty}(\lambda k+\lambda p+1-\lambda)(k+p)^{\Omega}\left\{k+p-p^{\Omega}(p-\alpha)(1+\lambda p-\lambda)\right\} a_{k+p} z^{k+p}\right|} \\
& \quad \frac{(1+\lambda p-\lambda) p^{\Omega}\left\{p^{\Omega}(p-\alpha)(1+\lambda p-\lambda)-p\right\}}{(1+\lambda p-\lambda) p^{\Omega} z^{p}|-| \sum_{k=n}^{\infty}(\lambda k+\lambda p+1-\lambda)(k+p)^{\Omega} a_{k+p} z^{k+p} \mid} \\
= & \frac{\left.\left|\sum_{k=n}^{\infty}(\lambda k+\lambda p+1-\lambda)(k+p)^{\Omega}\left\{k+p-p^{\Omega}(p-\alpha)(1+\lambda p-\lambda)\right\} a_{k+p}\right| z\right|^{k}}{(1+\lambda p-\lambda) p^{\Omega}-\sum_{k=n}^{\infty}(\lambda k+\lambda p+1-\lambda)(k+p)^{\Omega} a_{k+p}|z|^{k}}
\end{aligned}
$$




$$
\begin{aligned}
& (1+\lambda p-\lambda) p^{\Omega}\left\{p^{\Omega}(p-\alpha)(1+\lambda p-\lambda)-p\right\} \\
\leq & \frac{+\left\{p-p^{\Omega}(p-\alpha)(1+\lambda p-\lambda)\right\} \sum_{k=n}^{\infty}(\lambda k+\lambda p+1-\lambda)(k+p)^{\Omega} a_{k+p}}{(1+\lambda p-\lambda) p^{\Omega}-\sum_{k=n}^{\infty}(\lambda k+\lambda p+1-\lambda)(k+p)^{\Omega} a_{k+p}} \\
+ & \frac{\sum_{k=n}^{\infty} k(\lambda k+\lambda p+1-\lambda)(k+p)^{\Omega} a_{k+p}}{(1+\lambda p-\lambda) p^{\Omega}-\sum_{k=n}^{\infty}(\lambda k+\lambda p+1-\lambda)(k+p)^{\Omega} a_{k+p}} \\
\leq & \frac{\left\{p^{\Omega}(p-\alpha)(1+\lambda p-\lambda)-p\right\}\left\{(1+\lambda p-\lambda) p^{\Omega}-\sum_{k=n}^{\infty}(\lambda k+\lambda p+1-\lambda)(k+p)^{\Omega} a_{k+p}\right\}}{(1+\lambda p-\lambda) p^{\Omega}-\sum_{k=n}^{\infty}(\lambda k+\lambda p+1-\lambda)(k+p)^{\Omega} a_{k+p}} \\
+ & \frac{(1+\lambda p-\lambda) p^{\Omega}-\sum_{k=n}^{\infty}(\lambda k+\lambda p+1-\lambda)(k+p)^{\Omega} a_{k+p}}{(p-\alpha)(1+\lambda p-\lambda)-\sum_{k=n}^{\infty}(p-\alpha)(\lambda k+\lambda p+1-\lambda)(k+p)^{\Omega} a_{k+p}} \\
= & p^{\Omega}(p-\alpha)(1+\lambda p-\lambda)-p+p-\alpha \\
= & p^{\Omega}(p-\alpha)(1+\lambda p-\lambda)-\alpha \\
& \left(0 \leq \alpha<1 ; 0 \leq \lambda \leq 1 ; p \leq p^{\Omega}(p-\alpha)(1+\lambda p-\lambda)(p \neq 1) ;\right. \\
& \left.p \in \mathrm{N} ; n \in \mathrm{N} ; \Omega \in \mathrm{N}_{0}\right)
\end{aligned}
$$

provided that the inequality (2.1) is satisfied. Hence, by the maximum modulus theorem, we have

$$
f(z) \in T_{\Omega}(n, p, \lambda, \alpha)
$$

Finally, we note that the assertion (2.1) of Theorem 1 is sharp, the extremal function being

$$
\begin{aligned}
f(z)= & z^{p}-\frac{p^{\Omega}(p-\alpha)(1+\lambda p-\lambda)}{(k+p)^{\Omega}(k+p-\alpha)(\lambda k+\lambda p+1-\lambda)} z^{k+p} \\
& \left(k \geq n ; p, n \in \mathrm{N}, \Omega \in \mathrm{N}_{0}\right) .
\end{aligned}
$$

Corollary 1. Let the function $f(z)$ defined by (1.1) be in the class $T_{\Omega}(n, p, \lambda, \alpha)$. Then

$$
a_{k+p} \leq \frac{p^{\Omega}(p-\alpha)(1+\lambda p-\lambda)}{(k+p)^{\Omega}(k+p-\alpha)(\lambda k+\lambda p+1-\lambda)}(k \geq n) .
$$

The equality in (2.3) is attained for the function $f(z)$ given by (2.2). 


\section{Distortion theorems}

THEOREM 2. Let the function $f(z)$ defined by (1.1) be in the class $T_{\Omega}(n, p, \lambda, \alpha)$. Then we have

$$
|f(z)| \leq|z|^{p}+\frac{p^{\Omega}(p-\alpha)(1+\lambda p-\lambda)}{(n+p)^{\Omega}(n+p-\alpha)(\lambda p+\lambda n+1-\lambda)}|z|^{p+n}
$$

and

$$
|f(z)| \geq|z|^{p}-\frac{p^{\Omega}(p-\alpha)(1+\lambda p-\lambda)}{(n+p)^{\Omega}(n+p-\alpha)(\lambda p+\lambda n+1-\lambda)}|z|^{p+n}
$$

for $z \in U$. Then equalities in (3.1) and (3.2) are attained for the function $f(z)$ given by

$$
f(z)=z^{p}-\frac{p^{\Omega}(p-\alpha)(1+\lambda p-\lambda)}{(n+p)^{\Omega}(n+p-\alpha)(\lambda p+\lambda n+1-\lambda)} z^{p+n} .
$$

Proof. Note that

$$
\begin{aligned}
& (n+p)^{\Omega}(n+p-\alpha)(\lambda p+\lambda n+1-\lambda) \sum_{k=n}^{\infty} a_{k+p} \\
\leq & \sum_{k=n}^{\infty}(k+p)^{\Omega}(k+p-\alpha)(\lambda k+\lambda p+1-\lambda) a_{k+p} \\
\leq & p^{\Omega}(p-\alpha)(1+\lambda p-\lambda),
\end{aligned}
$$

this last inequality following from Theorem 1 . Thus

$$
\begin{aligned}
|f(z)| & \leq|z|^{p}+\sum_{k=n}^{\infty}\left|a_{k+p}\right||z|^{k+p} \\
& \leq|z|^{p}+|z|^{n+p} \sum_{k=n}^{\infty} a_{k+p} \\
& \leq|z|^{p}+\frac{p^{\Omega}(p-\alpha)(1+\lambda p-\lambda)}{(n+p)^{\Omega}(n+p-\alpha)(\lambda p+\lambda n+1-\lambda)}|z|^{p+n} .
\end{aligned}
$$

Similarly,

$$
\begin{aligned}
|f(z)| & \geq|z|^{p}-|z|^{n+p} \sum_{k=n}^{\infty} a_{k+p} \\
& \geq|z|^{p}-\frac{p^{\Omega}(p-\alpha)(1+\lambda p-\lambda)}{(n+p)^{\Omega}(n+p-\alpha)(\lambda p+\lambda n+1-\lambda)}|z|^{p+n} .
\end{aligned}
$$


THEOREM 3. If $f(z) \in T_{\Omega}(n, p, \lambda, \alpha)$, then

$$
\begin{aligned}
& |z|^{p-1}\left\{1-\frac{p^{\Omega-1}(p-\alpha)(1+\lambda p-\lambda)}{(n+p)^{\Omega-1}(n+p-\alpha)(\lambda p+\lambda n+1-\lambda)}|z|^{n}\right\} \\
\leq & \frac{1}{p}\left|f^{\prime}(z)\right| \\
\leq & |z|^{p-1}\left\{1+\frac{p^{\Omega-1}(p-\alpha)(1+\lambda p-\lambda)}{(n+p)^{\Omega-1}(n+p-\alpha)(\lambda p+\lambda n+1-\lambda)}|z|^{n}\right\} .
\end{aligned}
$$

Proof. We have

$$
\begin{aligned}
\left|f^{\prime}(z)\right| & \leq p|z|^{p-1}+\sum_{k=n}^{\infty}(k+p) a_{k+p}|z|^{k+p-1} \\
& \leq p|z|^{p-1}+|z|^{n+p-1} \sum_{k=n}^{\infty}(k+p) a_{k+p} .
\end{aligned}
$$

In view of Theorem 1 , we have

$$
\begin{aligned}
& \sum_{k=n}^{\infty}(k+p)^{\Omega}(k+p-\alpha)(\lambda k+\lambda p-\lambda+1) a_{k+p} \\
\leq & p^{\Omega}(p-\alpha)(1+\lambda p-\lambda)
\end{aligned}
$$

and then

$$
\begin{aligned}
& (n+p-\alpha)(\lambda n+\lambda p-\lambda+1)(n+p)^{\Omega-1} \sum_{k=n}^{\infty}(k+p) a_{k+p} \\
\leq & \sum_{k=n}^{\infty}(k+p)^{\Omega}(k+p-\alpha)(\lambda k+\lambda p-\lambda+1) a_{k+p} \\
\leq & p^{\Omega}(p-\alpha)(1+\lambda p-\lambda)
\end{aligned}
$$

or

$$
\begin{aligned}
& \sum_{k=n}^{\infty}(k+p) a_{k+p} \\
\leq & \frac{p^{\Omega}(p-\alpha)(1+\lambda p-\lambda)}{(n+p)^{\Omega-1}(n+p-\alpha)(\lambda p+\lambda n+1-\lambda)} .
\end{aligned}
$$

A substitution of (3.5) in to (3.4) yields the right-hand inequality. 
On the other hand,

$$
\begin{aligned}
\left|f^{\prime}(z)\right| & \geq p|z|^{p-1}-\sum_{k=n}^{\infty}(k+p) a_{k+p}|z|^{k+p-1} \\
& \geq p|z|^{p-1}-|z|^{n+p-1} \sum_{k=n}^{\infty}(k+p) a_{k+p} \\
& \geq p|z|^{p-1}-|z|^{n+p-1} \sum_{k=n}^{\infty}(k+p) a_{k+p} \\
& \geq p|z|^{p-1}-|z|^{n+p-1} \frac{p^{\Omega}(p-\alpha)(1+\lambda p-\lambda)}{(n+p)^{\Omega-1}(n+p-\alpha)(\lambda p+\lambda n+1-\lambda)}
\end{aligned}
$$

or

$$
\frac{\left|f^{\prime}(z)\right|}{p} \geq|z|^{p-1}\left\{1-\frac{p^{\Omega-1}(p-\alpha)(1+\lambda p-\lambda)}{(n+p)^{\Omega-1}(n+p-\alpha)(\lambda p+\lambda n-\lambda+1)}|z|^{n}\right\} .
$$

Corollary 2. Let the function $f(z)$ defined by (1.1) be in the class $T_{\Omega}(n, p, \lambda, \alpha)$. Then the unit disk $U$ is mapped onto a domain that contains the disk

$$
|w|<1-\left(\frac{p}{n+p}\right)^{\Omega}\left(\frac{p-\alpha}{n+p-\alpha}\right)\left(\frac{1+\lambda p-\lambda}{\lambda p+\lambda n+1-\lambda}\right) .
$$

The result is sharp with the extremal function given by (3.3).

\section{Closure theorems}

Let the functions $f_{j}(z)$ be defined, for $j=1,2, \ldots, m$ by

$$
f_{j}(z)=z^{p}-\sum_{k=n}^{\infty} a_{k+p, j} z^{k+p}\left(a_{k+p, j} \geq 0\right)
$$

for $z \in U[3]$.

We shall prove the following results for the closure of functions in the class $T_{\Omega}(n, p, \lambda, \alpha)$.

THEOREM 4. Let the functions $f_{j}(z)$ defined by $(4.1)$ be in the class $T_{\Omega}(n, p, \lambda, \alpha)$ for every $j=1,2, \ldots, m$. Then the functions $h(z)$ defined by

$$
h(z)=\sum_{j=1}^{m} c_{j} f_{j}(z)\left(c_{j} \geq 0\right)
$$


is also in the same class $T_{\Omega}(n, p, \lambda, \alpha)$, where

$$
\sum_{j=1}^{m} c_{j}=1
$$

Proof. According to the definition of $h(z)$, we can write

$$
\begin{aligned}
h(z) & =\sum_{j=1}^{m} c_{j}\left[z^{p}-\sum_{k=n}^{\infty} a_{k+p, j} z^{k+p}\right] \\
& =\left(\sum_{j=1}^{m} c_{j}\right) z^{p}-\sum_{k=n}^{\infty}\left(\sum_{j=1}^{m} c_{j} a_{k+p, j}\right) z^{k+p} \\
& =z^{p}-\sum_{k=n}^{\infty}\left(\sum_{j=1}^{m} c_{j} a_{k+p, j}\right) z^{k+p} .
\end{aligned}
$$

Further, since $f_{j}(z)$ are in $T_{\Omega}(n, p, \lambda, \alpha)$ for every $j=1,2, \ldots, m$ we get

$$
\begin{aligned}
& \sum_{k=n}^{\infty}(k+p)^{\Omega}(k+p-\alpha)(\lambda k+\lambda p-\lambda+1) a_{k+p, j} \\
\leq & p^{\Omega}(p-\alpha)(1+\lambda p-\lambda)
\end{aligned}
$$

for every $j=1,2, \ldots, m$. Hence we can see that

$$
\begin{aligned}
& \sum_{k=n}^{\infty}(k+p)^{\Omega}(k+p-\alpha)(\lambda k+\lambda p-\lambda+1)\left(\sum_{j=1}^{m} c_{j} a_{k+p, j}\right) \\
= & \sum_{k=n}^{\infty}(k+p)^{\Omega}(k+p-\alpha)(\lambda k+\lambda p-\lambda+1) \\
\times & \left(c_{1} a_{k+p, 1}+c_{2} a_{k+p, 2}+\ldots+c_{m} a_{k+p, m}\right) \\
= & c_{1} \sum_{k=n}^{\infty}(k+p)^{\Omega}(k+p-\alpha)(\lambda k+\lambda p-\lambda+1) a_{k+p, 1} \\
& +c_{2} \sum_{k=n}^{\infty}(k+p)^{\Omega}(k+p-\alpha)(\lambda k+\lambda p-\lambda+1) a_{k+p, 2}+\cdots \\
& +c_{m} \sum_{k=n}^{\infty}(k+p)^{\Omega}(k+p-\alpha)(\lambda k+\lambda p-\lambda+1) a_{k+p, m}
\end{aligned}
$$




$$
\begin{aligned}
\leq & c_{1}\left[p^{\Omega}(p-\alpha)(1+\lambda p-\lambda)\right]+c_{2}\left[p^{\Omega}(p-\alpha)(1+\lambda p-\lambda)\right]+\cdots \\
& +c_{m}\left[p^{\Omega}(p-\alpha)(1+\lambda p-\lambda)\right] \\
= & \left(c_{1}+c_{2}+\ldots+c_{m}\right)\left[p^{\Omega}(p-\alpha)(1+\lambda p-\lambda)\right] \\
= & \left(\sum_{j=1}^{m} c_{j}\right) p^{\Omega}(p-\alpha)(1+\lambda p-\lambda) \\
= & p^{\Omega}(p-\alpha)(1+\lambda p-\lambda)
\end{aligned}
$$

which implies that $h(z)$ in $T_{\Omega}(n, p, \lambda, \alpha)$. Thus we have the theorem.

Corollary 3. Let the function $f(z)$ defined by (1.1) and the function $g(z)$ defined by

$$
g(z)=z^{p}-\sum_{k=n}^{\infty} b_{k+p} z^{k+p}\left(b_{k+p} \geq p \in \mathrm{N} n \in \mathrm{N}\right)
$$

be in the same class $T_{\Omega}(n, p, \lambda, \alpha)$. Then the function $h(z)$ defined by

$$
\begin{aligned}
h(z)= & (1-\gamma) f(z)+\gamma g(z) \\
= & z^{p}-\sum_{k=n}^{\infty} c_{k+p} z^{k+p} \\
& \left(c_{k+p} \geq 0 ; 0 \leq \gamma \leq 1 ; p \in \mathrm{N} ; n \in \mathrm{N}\right)
\end{aligned}
$$

is also in the class $T_{\Omega}(n, p, \lambda, \alpha)$.

Proof. Suppose that each of the functions $f(z)$ and $g(z)$ is in the class $T_{\Omega}(n, p, \lambda, \alpha)$. Then making use of (2.1), we see that

$$
\begin{aligned}
& \sum_{k=n}^{\infty}(k+p)^{\Omega}(k+p-\alpha)(\lambda k+\lambda p-\lambda+1) c_{k+p} \\
= & (1-\gamma) \sum_{k=n}^{\infty}(k+p)^{\Omega}(k+p-\alpha)(\lambda k+\lambda p-\lambda+1) a_{k+p} \\
& +\gamma \sum_{k=n}^{\infty}(k+p)^{\Omega}(k+p-\alpha)(\lambda k+\lambda p-\lambda+1) b_{k+p} \\
\leq & (1-\gamma) p^{\Omega}(p-\alpha)(1+\lambda p-\lambda)+\gamma(p-\alpha) p^{\Omega}(1+\lambda p-\lambda) \\
= & p^{\Omega}(p-\alpha)(1+\lambda p-\lambda) \\
& \left(0 \leq \alpha<1,0 \leq \lambda \leq 1, p \leq p^{\Omega}(p-\alpha)(1+\lambda p-\lambda)(p \neq 1) ;\right. \\
& \left.p \in \mathrm{N}, n \in \mathrm{N} ; \Omega \in \mathrm{N}_{0}\right),
\end{aligned}
$$

which of the completes the proof of Corollary 3 . 
As a consequence of Corollary 3 , there exists the extreme points of the class $T_{\Omega}(n, p, \lambda, \alpha)$.

TheOREM 5. Let $f_{n-1}(z)=z^{p}$ and

$$
f_{k}(z)=z^{p}-\frac{p^{\Omega}(p-\alpha)(1+\lambda p-\lambda)}{(k+p)^{\Omega}(k+p-\alpha)(\lambda k+\lambda p-\lambda+1)} z^{k+p}, \quad(k \geq n)
$$

for $0 \leq \alpha<1,0 \leq \lambda \leq 1$ and $n \in \mathrm{N}$. Then $f(z)$ is in the class $T_{\Omega}(n, p, \lambda, \alpha)$ if and only if it can be expressed in the form

$$
f(z)=\sum_{k=n-1}^{\infty} \eta_{k} f_{k}(z)
$$

where $\eta_{k} \geq 0,(k \geq n-1)$ and $\sum_{k=n-1}^{\infty} \eta_{k}=1$.

Proof. Suppose that

$$
f(z)=\sum_{k=n-1}^{\infty} \eta_{k} f_{k}(z) .
$$

Then

$$
\begin{aligned}
f(z) & =\sum_{k=n-1}^{\infty} \eta_{k} f_{k}(z)=\eta_{n-1} f_{n-1}(z)+\sum_{k=n}^{\infty} \eta_{k} f_{k}(z) \\
& =\eta_{n-1} z^{p}+\sum_{k=n}^{\infty} \eta_{k}\left[z^{p}-\frac{p^{\Omega}(p-\alpha)(1+\lambda p-\lambda)}{(k+p)^{\Omega}(p+k-\alpha)(\lambda k+\lambda p-\lambda+1)}\right] z^{k+p} \\
& =\left(\sum_{k=n-1}^{\infty} \eta_{k}\right) z^{p}-\sum_{k=n}^{\infty} \eta_{k} \frac{p^{\Omega}(p-\alpha)(1+\lambda p-\lambda)}{(k+p)^{\Omega}(p+k-\alpha)(\lambda k+\lambda p-\lambda+1)} z^{k+p} \\
& =z^{p}-\sum_{k=n}^{\infty} \frac{p^{\Omega}(p-\alpha)(1+\lambda p-\lambda)}{(k+p)^{\Omega}(p+k-\alpha)(\lambda k+\lambda p-\lambda+1)} \eta_{k} z^{k+p} .
\end{aligned}
$$

Thus

$$
\begin{aligned}
& \sum_{k=n}^{\infty} \eta_{k} {\left[\frac{p^{\Omega}(p-\alpha)(1+\lambda p-\lambda)}{(k+p)^{\Omega}(p+k-\alpha)(\lambda k+\lambda p-\lambda+1)}\right] } \\
& \times\left[\frac{(k+p)^{\Omega}(k+p-\alpha)(\lambda k+\lambda p-\lambda+1)}{p^{\Omega}(p-\alpha)(1+\lambda p-\lambda)}\right] \\
&=\sum_{k=n}^{\infty} \eta_{k}=\sum_{k=n-1}^{\infty} \eta_{k}-\eta_{n-1}=1-\eta_{n-1} \leq 1,
\end{aligned}
$$

so by Theorem $1, f(z) \in T_{\Omega}(n, p, \lambda, \alpha)$. 
Conversely, suppose $f(z) \in T_{\Omega}(n, p, \lambda, \alpha)$. Since

$$
a_{k+p} \leq \frac{p^{\Omega}(p-\alpha)(1+\lambda p-\lambda)}{(k+p)^{\Omega}(k+p-\alpha)(\lambda k+\lambda p-\lambda+1)} \quad(k=n, n+1, \ldots),
$$

we may set

$$
\eta_{k}=\frac{(k+p)^{\Omega}(k+p-\alpha)(\lambda k+\lambda p-\lambda+1)}{p^{\Omega}(p-\alpha)(1+\lambda p-\lambda)} a_{k+p}
$$

and

$$
\eta_{n-1}=1-\sum_{k=n}^{\infty} \eta_{k}
$$

Then

$$
\begin{aligned}
f(z) & =z^{p}-\sum_{k=n}^{\infty} a_{k+p} z^{k+p} \\
& =z^{p}-\sum_{k=n}^{\infty} \frac{p^{\Omega}(p-\alpha)(1+\lambda p-\lambda)}{(k+p)^{\Omega}(p+k-\alpha)(\lambda k+\lambda p-\lambda+1)} \eta_{k} z^{k+p} \\
& =z^{p}-\sum_{k=n}^{\infty} \eta_{k}\left[z^{p}-f_{k}(z)\right] \\
& =z^{p}-\sum_{k=n}^{\infty} \eta_{k} z^{p}+\sum_{k=n}^{\infty} \eta_{k} f_{k}(z) \\
& =\left(1-\sum_{k=n}^{\infty} \eta_{k}\right) z^{p}+\sum_{k=n}^{\infty} \eta_{k} f_{k}(z) \\
& =\eta_{n-1} z^{p}+\sum_{k=n}^{\infty} \eta_{k} f_{k}(z) \\
& =\eta_{n-1} f_{n-1}(z)+\sum_{k=n}^{\infty} \eta_{k} f_{k}(z) \\
& =\sum_{k=n-1}^{\infty} \eta_{k} f_{k}(z) .
\end{aligned}
$$

This completes the proof. 


\section{Integral operators}

Theorem 6. Let the function $f(z)$ defined by (1.1) be in the class $T_{\Omega}(n, p, \lambda, \alpha)$ and let $c$ be real number such that $c>-p$. Then the function $F(z)$ defined by

$$
F(z)=\frac{c+p}{z^{c}} \int_{0}^{z} t^{c-1} f(t) d t
$$

also belongs to the class $T_{\Omega}(n, p, \lambda, \alpha)$.

Proof. From the representation of $F(z)$, it follows that

$$
F(z)=z^{p}-\sum_{k=n}^{\infty} b_{k+p} z^{k+p},
$$

where

Therefore,

$$
b_{k+p}=\left(\frac{c+p}{c+p+k}\right) a_{k+p} .
$$

$$
\begin{aligned}
& \sum_{k=n}^{\infty}(k+p)^{\Omega}(k+p-\alpha)(\lambda k+\lambda p-\lambda+1) b_{k+p} \\
= & \sum_{k=n}^{\infty}(k+p)^{\Omega}(k+p-\alpha)(\lambda k+\lambda p-\lambda+1)\left(\frac{c+p}{c+p+k}\right) a_{k+p} \\
\leq & \sum_{k=n}^{\infty}(k+p)^{\Omega}(k+p-\alpha)(\lambda k+\lambda p-\lambda+1) a_{k+p} \\
\leq & p^{\Omega}(p-\alpha)(1+\lambda p-\lambda),
\end{aligned}
$$

since $f(z) \in T_{\Omega}(n, p, \lambda, \alpha)$. Hence, by Theorem $1, F(z) \in T_{\Omega}(n, p, \lambda, \alpha)$.

THEOREM 7. Let $c$ be real number such that $c>-p$. If $F(z) \in$ $T_{\Omega}(n, p, \lambda, \alpha)$, then the function $f(z)$ defined by (5.1) is $p$-valent in $|z|<R_{p}^{*}$, where

$$
\begin{gathered}
R_{p}^{*}=\inf _{k}\left\{\left(\frac{k+p}{p}\right)^{\Omega-1}\left(\frac{c+p}{c+p+k}\right)\left(\frac{k+p-\alpha}{p-\alpha}\right)\right. \\
\left.\left(\frac{\lambda k+\lambda p-\lambda+1}{\lambda p-\lambda+1}\right)\right\}^{\frac{1}{k}}(k \geq n) .
\end{gathered}
$$

The result is sharp. 
Proof. Let

$$
F(z)=z^{p}-\sum_{k=n}^{\infty} a_{k+p} z^{k+p} \quad\left(a_{k+p} \geq 0\right) .
$$

It follow from (5.1) that

$$
\begin{aligned}
f(z) & =\frac{z^{1-c}\left(z^{c} F(z)\right)^{\prime}}{(c+p)} \quad(c>-p) \\
& =z^{p}-\sum_{k=n}^{\infty}\left(\frac{c+k+p}{c+p}\right) a_{k+p} z^{k+p} .
\end{aligned}
$$

To prove the result, it suffices to show that

$$
\left|\frac{f^{\prime}(z)}{z^{p-1}}-p\right| \leq p \text { for }|z|<R_{p}^{*}
$$

Now

$$
\begin{aligned}
& \quad\left|\frac{f^{\prime}(z)}{z^{p-1}}-p\right|=\left|\frac{p z^{p-1}-\sum_{k=n}^{\infty}(k+p)\left(\frac{c+k+p}{c+p}\right) a_{k+p} z^{k+p-1}}{z^{p-1}}-p\right| \\
& \left|-\sum_{k=n}^{\infty}(k+p)\left(\frac{c+p+k}{c+p}\right) a_{k+p} z^{k}\right| \leq \sum_{k=n}^{\infty}(k+p)\left(\frac{c+p+k}{c+p}\right) a_{k+p}|z|^{k} . \\
& \text { Thus }\left|\frac{f^{\prime}(z)}{z^{p-1}}-p\right| \leq p \text { if } \\
& \text { (5.3) } \quad \sum_{k=n}^{\infty}\left(\frac{k+p}{p}\right)\left(\frac{c+p+k}{c+p}\right) a_{k+p}|z|^{k} \leq 1 .
\end{aligned}
$$

But Theorem 1 confirms that

$$
\sum_{k=n}^{\infty} \frac{(k+p)^{\Omega}(k+p-\alpha)(\lambda k+\lambda p-\lambda+1)}{p^{\Omega}(p-\alpha)(1+\lambda p-\lambda)} a_{k+p} \leq 1 .
$$

Thus (5.3) will be satisfied if

$$
\begin{aligned}
& \left(\frac{k+p}{p}\right)\left(\frac{c+p+k}{c+p}\right)|z|^{k} \\
\leq & \frac{(k+p)^{\Omega}}{p^{\Omega}}\left(\frac{k+p-\alpha}{p-\alpha}\right)\left(\frac{\lambda k+\lambda p-\lambda+1}{1+\lambda p-\lambda}\right) \quad(k \geq n),
\end{aligned}
$$


or if

$$
\begin{aligned}
|z| \leq\{ & \left(\frac{k+p}{p}\right)^{\Omega-1}\left(\frac{c+p}{c+p+k}\right) \\
& \left.\left(\frac{k+p-\alpha}{p-\alpha}\right)\left(\frac{\lambda k+\lambda p-\lambda+1}{\lambda p-\lambda+1}\right)\right\}^{\frac{1}{k}}(k \geq n) .
\end{aligned}
$$

The required result follows now from (5.5). The result is sharp for the function

$$
\begin{aligned}
f(z) & =z^{p}-\frac{p^{\Omega}(p-\alpha)(1+\lambda p-\lambda)}{(k+p)^{\Omega}(k+p-\alpha)(\lambda k+\lambda p-\lambda+1)} \\
& \times\left(\frac{c+p+k}{c+p}\right) z^{k+p} \quad(k \geq n) .
\end{aligned}
$$

THEOREM 8. Let the function $f(z)$ defined by (1.1) be in the class $T_{\Omega}(n, p, \lambda, \alpha)$. Then $f(z)$ is convex of order $q(0 \leq q<1)$ in $|z|<r$, where

$$
\begin{aligned}
r=\inf _{k}\{ & \left(\frac{k+p}{p}\right)^{\Omega-1}\left(\frac{p-q}{p-\alpha}\right) \\
& \left.\left(\frac{k+p-\alpha}{k+p-q}\right)\left(\frac{\lambda k+\lambda p-\lambda+1}{\lambda p-\lambda+1}\right)\right\}^{\frac{1}{k}}(k \geq n) .
\end{aligned}
$$

Proof. We must show that

$$
\left|\frac{z f^{\prime \prime}(z)}{f^{\prime}(z)}+1-p\right|<p-q
$$

$(0 \leq q<1)$ for $|z|<r$. We have

$$
\begin{aligned}
&\left|\frac{z f^{\prime \prime}(z)}{f^{\prime}(z)}+1-p\right|=\left|\frac{z f^{\prime \prime}(z)+(1-p) f^{\prime}(z)}{f^{\prime}(z)}\right| \\
& \mid \frac{p(p-1) z^{p-1}-\sum_{k=n}^{\infty}(k+p)(k+p-1) a_{k+p} z^{k+p-1}}{p z^{p-1}-\sum_{k=n}^{\infty}(k+p) a_{k+p} z^{k+p-1}} \\
&-\frac{(p-1) p z^{p-1}+\sum_{k=n}^{\infty}(p-1)(k+p) a_{k+p} z^{k+p-1}}{p z^{p-1}-\sum_{k=n}^{\infty}(k+p) a_{k+p} z^{k+p-1}} \mid
\end{aligned}
$$




$$
\begin{aligned}
& =\left|\frac{-\sum_{k=n}^{\infty}(k+p) k a_{k+p} z^{k+p-1}}{p z^{p-1}-\sum_{k=n}^{\infty}(k+p) a_{k+p} z^{k+p-1}}\right| \\
& \leq \frac{\sum_{k=n}^{\infty} k(k+p) a_{k+p}|z|^{k}}{p-\sum_{k=n}^{\infty}(k+p) a_{k+p}|z|^{k}} .
\end{aligned}
$$

Thus $\left|\frac{z f^{\prime \prime}(z)}{f^{\prime}(z)}+1-p\right|<p-q$ if

$$
\sum_{k=n}^{\infty} \frac{(k+p)(k+p-q)}{p(p-q)} a_{k+p}|z|^{k} \leq 1 .
$$

But Theorem 1 confirms that

$$
\sum_{k=n}^{\infty} \frac{(k+p)^{\Omega}(k+p-\alpha)(\lambda k+\lambda p-\lambda+1)}{p^{\Omega}(p-\alpha)(1+\lambda p-\lambda)} a_{k+p} \leq 1 .
$$

Hence (5.7) will be true if

$$
\frac{(k+p)(k+p-q)}{p(p-q)}|z|^{k} \leq \frac{(k+p)^{\Omega}(k+p-\alpha)(\lambda k+\lambda p-\lambda+1)}{p^{\Omega}(p-\alpha)(1+\lambda p-\lambda)}
$$

or if

$$
\begin{gathered}
|z|^{k} \leq \frac{(k+p)^{\Omega}(k+p-\alpha)(\lambda k+\lambda p-\lambda+1) p(p-q)}{p^{\Omega}(p-\alpha)(1+\lambda p-\lambda)(k+p-q)(k+p)} \\
|z| \leq\left\{\left(\frac{k+p}{p}\right)^{\Omega-1}\left(\frac{p-q}{p-\alpha}\right)\left(\frac{k+p-\alpha}{k+p-q}\right)\right. \\
\left.\left(\frac{\lambda k+\lambda p-\lambda+1}{1+\lambda p-\lambda}\right)\right\}^{\frac{1}{k}}(k \geq n) .
\end{gathered}
$$

\section{Modified Hadamard products}

Let the function $f(z)$ defined by (1.1) and the function $g(z)$ defined by

$$
g(z)=z^{p}-\sum_{k=n}^{\infty} b_{k+p} z^{k+p}\left(b_{k+p} \geq 0 ; p \in \mathrm{N}, n \in \mathrm{N}\right)
$$


be in the same class $T_{\Omega}(n, p, \lambda, \alpha)$. We define the modified Hadamard product of the functions $f(z)$ and $g(z)$ by

$$
f * g(z)=z^{p}-\sum_{k=n}^{\infty} a_{k+p} b_{k+p} z^{k+p} .
$$

THEOREM 9. If each of the functions $f(z)$ and $g(z)$ is in the class $T_{\Omega}(n, p, \lambda, \alpha)$, then

$$
f * g(z) \in T_{\Omega}(n, p, \lambda, \delta),
$$

where

(6.1)

$\delta \leq p-n$

$$
\begin{aligned}
& \times \frac{p^{\Omega}(p-\alpha)^{2}(1+\lambda p-\lambda)}{(n+p)^{\Omega}(n+p-\alpha)^{2}(\lambda n+\lambda p-\lambda+1)-p^{\Omega}(p-\alpha)^{2}(1+\lambda p-\lambda)} \\
& \quad(p \in \mathrm{N}, n \in \mathrm{N}) .
\end{aligned}
$$

The result is sharp for the functions $f(z)$ and $g(z)$ given by

$$
\begin{aligned}
f(z)= & g(z) \\
= & z^{p}-\frac{p^{\Omega}(p-\alpha)(1+\lambda p-\lambda)}{(n+p)^{\Omega}(n+p-\alpha)(\lambda n+\lambda p-\lambda+1)} z^{n+p} \\
& (p \in \mathrm{N}, n \in \mathrm{N}) .
\end{aligned}
$$

Proof. Employing the technique used earlier by Schild and Silverman [6], we need to find the largest $\delta$ such that

$$
\sum_{k=n}^{\infty} \frac{(k+p)^{\Omega}(k+p-\delta)(\lambda k+\lambda p-\lambda+1)}{p^{\Omega}(p-\delta)(1+\lambda p-\lambda)} a_{k+p} b_{k+p} \leq 1 .
$$

Since

$$
\sum_{k=n}^{\infty} \frac{(k+p)^{\Omega}(k+p-\alpha)(\lambda k+\lambda p-\lambda+1)}{p^{\Omega}(p-\alpha)(1+\lambda p-\lambda)} a_{k+p} \leq 1
$$

and

$$
\sum_{k=n}^{\infty} \frac{(k+p)^{\Omega}(k+p-\alpha)(\lambda k+\lambda p-\lambda+1)}{p^{\Omega}(p-\alpha)(1+\lambda p-\lambda)} b_{k+p} \leq 1,
$$

by the Cauchy-Schwarz inequality, we have

$$
\sum_{k=n}^{\infty} \frac{(k+p)^{\Omega}(k+p-\alpha)(\lambda k+\lambda p-\lambda+1)}{p^{\Omega}(p-\alpha)(1+\lambda p-\lambda)} \sqrt{a_{k+p} b_{k+p}} \leq 1 .
$$


Thus it is sufficient to show that

$$
\begin{aligned}
& \frac{(k+p)^{\Omega}(k+p-\delta)(\lambda k+\lambda p-\lambda+1)}{p^{\Omega}(p-\delta)(1+\lambda p-\lambda)} a_{k+p} b_{k+p} \\
\leq & \frac{(k+p)^{\Omega}(k+p-\alpha)(\lambda k+\lambda p-\lambda+1)}{p^{\Omega}(p-\alpha)(1+\lambda p-\lambda)} \sqrt{a_{k+p} b_{k+p}}
\end{aligned}
$$

that is, that

$$
\sqrt{a_{k+p} b_{k+p}} \leq \frac{(k+p-\alpha)}{(k+p-\delta)} \cdot \frac{(p-\delta)}{(p-\alpha)}
$$

Not that

$$
\sqrt{a_{k+p} b_{k+p}} \leq \frac{p^{\Omega}(p-\alpha)(1+\lambda p-\lambda)}{(k+p)^{\Omega}(k+p-\alpha)(\lambda k+\lambda p-\lambda+1)} \quad(k \geq n) .
$$

Consequently, we need only to prove that

$$
\frac{p^{\Omega}(p-\alpha)(1+\lambda p-\lambda)}{(k+p)^{\Omega}(k+p-\alpha)(\lambda k+\lambda p-\lambda+1)} \leq \frac{k+p-\alpha}{k+p-\delta} \frac{p-\delta}{p-\alpha} \quad(k \geq n),
$$

or, equivalently, that

$$
\delta \leq p
$$

$$
-\frac{p^{\Omega}(p-\alpha)^{2}(1+\lambda p-\lambda)}{(k+p)^{\Omega}(k+p-\alpha)^{2}(\lambda k+\lambda p-\lambda+1)-p^{\Omega}(p-\alpha)^{2}(1+\lambda p-\lambda)} k
$$

$(k \geq n)$.

Since

$$
\begin{aligned}
& \psi(k) \\
= & p-\frac{p^{\Omega}(p-\alpha)^{2}(1+\lambda p-\lambda)}{(k+p)^{\Omega}(k+p-\alpha)^{2}(\lambda k+\lambda p-\lambda+1)-p^{\Omega}(p-\alpha)^{2}(1+\lambda p-\lambda)} k \\
& (k \geq n) .
\end{aligned}
$$

is an increasing function of $k \quad(k \geq n)$, letting $k=n(6.2)$, we obtain $\delta \leq \psi(n)$

$$
=p-\frac{p^{\Omega}(p-\alpha)^{2}(1+\lambda p-\lambda)}{(n+p)^{\Omega}(n+p-\alpha)^{2}(\lambda n+\lambda p-\lambda+1)-p^{\Omega}(p-\alpha)^{2}(1+\lambda p-\lambda)} n,
$$

which completes the proof Theorem 9 .

Finally, by taking the function $f_{j}(z)$ given by

$$
f_{j}(z)=z^{p}-\frac{p^{\Omega}(p-\alpha)(1+\lambda p-\lambda)}{(n+p)^{\Omega}(n+p-\alpha)(\lambda n+\lambda p-\lambda+1)} z^{n+p} \quad(j=1,2),
$$

we can see that the result is sharp. 


\section{References}

[1] O. Altıntaş, On a subclass of certain starlike functions with negative coefficients, Math. Japon. 36 (1991), no. 3, 489-495.

[2] O. Altintaş, H. Irmak and H. M. Srivastava, Fractional calculus and certain starlike functions with negative coefficients, Comput. Math. Appl. 30 (1995), no. 2, 9-15.

[3] M. K. Aouf, A. Shamandy and A. A. Att1y1a, Certain classes of analytic and multivalent functions with negative coefficients, Turkish J. Math. 20 (1996), no. $3,353-368$.

[4] M. Kamali and S. Akbulut, On a subclass of certain convex functions with negative coefficients, Appl. Math. Comput. 145 (2003), no. 2-3, 341-350.

[5] G. S. Salagean, Subclasses of univalent functions, Lecture Notes in Math. SpringerVerlag 1013 (1983), 362-372.

[6] A. Schild and H. Silverman, Convolutions of univalent functions with negative coefficients, Ann. Univ. Mariae Curie-Sklodowska Sect. A 29 (1975), 99-107.

[7] H. Silverman, Univalent functions with negative coefficients, Proc. Amer. Math. Soc. 51 (1975), 109-116.

[8] H. M. Srivastava, S. Owa and S. K. Chatterjea, A note on certain classes of starlike functions, Rend. Sem. Math. Univ. Padova 77 (1987), 115-124.

Atatürk Üniversitesi, Fen-Edebiyat FakÜltesi, Matematik Bölümü, 25240 ERZURUM, TURKEY

E-mail: mkamali@atauni.edu.tr 